\title{
Investigation of Neutrino-Nucleon Interaction through Intermediate Vector Boson (IVB)
}

\author{
Mohamed Tarek Hussein ${ }^{1}$, Ahmad Islam Saad ${ }^{2}$ \\ ${ }^{1}$ Physics Department, Faculty of Science, Cairo University, Giza, Egypt \\ ${ }^{2}$ Physics and Chemistry Department, Faculty of Education, Alexandria University, Alexandria, Egypt \\ E-mail: tarek@cu.edu.eg \\ Received July 15, 2010; revised August 18, 2010; accepted August 12, 2010
}

\begin{abstract}
This work deals with the interaction of neutrino with the nucleon considering data taken from different experiments. It is assumed that the interaction of neutrino with nucleons go through the intermediate vector boson (IVB) which may be the $\mathrm{W}$ or $\mathrm{Z}$ with effective mass of the order of $80 \mathrm{GeV}$. The neutrino wave function is obtained via perturbation technique to calculate the weak leptonic current. On the other hand, the quark current is estimated using the measured experimental data of deep inelastic scattering of neutrino-nucleon interaction. Eventually the total interaction transition matrix is calculated as a function of momentum transfer square, $q^{2}$ and qualitatively compared with the available experimental data. Besides, a comparative study is also done to explore the influence of the target composition during the neutrino weak interactions. In this context an investigation of neutrino-proton and neutrino-neutron interactions are carried out to calculate the deep inelastic cross section in both cases.
\end{abstract}

Keywords: Deep Inelastic Scattering (DIS), Intermediate Vector Boson (IVB), Parton Model, Structure Function

\section{Introduction}

The problem of weak interactions through the charged and neutral currents is dealt by many different approaches. A classical picture of lepton neutral current by James L. Carr [1] considered that, when charged current weak interactions are excluded, the neutral current weak interaction is formally similar to ordinary electromagnetism with a massive photon. In this spirit, the Maxwell equations for the fields of the Z-boson are derived from the standard model. For neutral current events, electrons (or neutrinos) remain as electrons (or neutrinos).

In the charged current case, an initial electron state emerges as a final neutrino state or vice-versa. For this reason it is difficult to consider such a picture for the charged current interaction. A non-relativistic weak-field Hamiltonian for the electron is developed which allows computing the interaction energy of an electron in the presence of a classical Z-boson field. The Maxwell equations for the Z-boson are then developed. In the absence of sources, the Maxwell equations, [2] are identical to those of ordinary electromagnetism but with a massive photon. The Maxwell equation source terms are derived from the interaction energies for both electron and neu- trino sources. The Maxwell equations derived in this case can be used to describe the Z-boson field generated by macroscopic or atomic-scale. They may also be used to visualize the Z-boson fields surrounding classical pointlike electrons and neutrinos. The classical point particle solutions provide an interesting visualization of the parity violation in the standard model in terms of a vortex-like magnetic field structure oriented with the electron's spin.

In calculating the cross section of neutrino nucleon interactions, we consider the three independent helicity states $(-1,+1,0)$ for the mediating bosons $\mathrm{W}^{ \pm}$. In the weak interactions there is no conservation of parity which compels helicity -1 and +1 states to occur with equal probability as a coherent superposition as in electromagnetic case. Thus for e-nucleon interactions we need 2-structure functions $\left(\mathrm{F}_{1}\right.$ and $\left.\mathrm{F}_{2}\right)$ to describe the inelastic cross section while 3 -structure functions $\left(\mathrm{F}_{1}, \mathrm{~F}_{2}\right.$ and $\mathrm{F}_{3}$ ) are needed for neutrino nucleon interactions.

\section{Background}

An alternative method was developed by T. Siiskonen et al., [3], where the phenomenological structure of the 
weak hadronic current between the proton and neutron states is well determined by its properties under the Lorentz transformation. Additional constraints come from the requirement of time reversal symmetry as well as from the invariance under the G-parity transformation (combined charge conjugation and isospin rotation). The resulting interaction Hamiltonian consists of vector $(\mathrm{V})$, axial vector $(\mathrm{A})$, induced weak magnetism $(\mathrm{M})$, and induced pseudoscalar $(\mathrm{P})$ terms together with the associated form factors $C_{\alpha}, \alpha=V, A, M$, or P. These form factors are called as coupling constants at zero momentum transfer. The present experimental knowledge does not exclude the presence of the scalar and tensor interactions. However, their contribution is expected to be small due to weak coupling [4]. The values of vector, axial vector, and weak magnetism couplings are well established by beta-decay experiments as well as by the conserved vector current hypothesis (CVC), introduced already in the late 50's [5]. The magnitude of the pseudoscalar coupling is more uncertain, although the partially-conserved axial current hypothesis (PCAC) [6] provides an estimate along with muon capture experiments in hydrogen $[7,8]$. In nuclear beta decay, with an energy release up to some $20 \mathrm{MeV}$, only the vector (Fermi) and the axial vector (Gamow-Teller) terms are usually important. The induced pseudoscalar and weak magnetism parts are essentially inactive, since their contributions are proportional to $\mathrm{q} / \mathrm{M}$, where $\mathrm{q}$ is the energy release and $\mathrm{M}$ is the nucleon mass (in units where $\hbar=\mathrm{c}=1$ ).

T. Siiskonen et al., [9,10] constructed effective operators for the weak hadronic current between proton and neutron states. These operators take into account the core polarization effects, which are expected to be the largest correction to the bare matrix element [11].

As mentioned earlier, Fermi conceived of $\beta$-decay as a process analogous to that of an electromagnetic transition, the electron-neutrino $(e-v)$ pair are playing the role of the emitted photon. The amplitude was assumed to involve, for the nucleons, the hadronic weak current matrix element $\left\langle\mathrm{p}\left|\mathrm{J}_{\mu}\right| \mathrm{n}>\right.$, in analogy to the electromagnetic transition currents. A simple Lorentz invariant amplitude is then obtained if ev pair also appears as a 4-vector combination $<\mathrm{ev}\left|\mathrm{J}^{\mu}\right| \mathrm{O}>$, which is the leptonic weak current matrix element. The complete matrix element being, $\mathrm{M}=$ $<\mathrm{p}\left|\mathrm{J}_{\mu}\right| \mathrm{n}><\mathrm{ev}\left|\mathrm{J}^{\mu}\right| \mathrm{O}>$. At very low energy release, one might expect that, to a good approximation, all momentum dependence in the matrix element could be ignored, reducing it to a constant $\mathrm{G}=1.14 \times 10^{-5} \mathrm{GeV}^{-2}$, in the natural units $(\hbar=\mathrm{c}=1)$. The first statement of universality of weak interactions was that all processes have the same coupling constant G. Fermi's vector-vector theory was motivated by the analogy of the vector currents of QED. The analogy was however, imperfect. The photon emitted in a radioactive transition is the quantum of the electromagnetic field, but it is hard to see how the corresponding e $v$ pair can be the weak field quantum, since the effective mass of the pair varies from process to another. It is therefore natural to postulate the existence of a weak analogue of the photon-the intermediate vector boson (IVB) and to suppose that weak interactions are mediated by the exchange of IVB's as the electromagnetic ones are by photon exchange. This was the first step toward an eventual unification at the weak and electromagnetic fields. In the presence of currents, the wave equation for the photon has the form:

$$
A^{\mu}=j_{e m}^{\mu}
$$

The propagator associated to the process is just the inverse of the differential operator in Equation (1). Applying this to the free particle, we get $-q^{2} A^{\mu}=j_{e m}^{\mu}$, hence the propagator is $-g^{\mu v} / q^{2}$. As for a massive spin-1 particle, in a general gauge, the Maxwell equations read

$$
A^{\mu}-\partial^{\mu} \partial^{v} A_{v}=j^{\mu}
$$

We make the natural replacement $\square \Rightarrow\left(\square+\mathrm{M}^{2}\right)$ to get,

$$
\left(\square+M^{2}\right) W^{\mu}-\partial^{\mu} \partial^{v} W_{v}=j^{\mu}
$$

For plane-wave solution,

$\left[\left(-q^{2}+M^{2}\right) g^{\mu v}+q^{\mu} q^{v}\right] W_{v}=j^{\mu}$. And the propagator is expected in this case to correspond to the inverse operator $\left[\left(-q^{2}+M^{2}\right) g^{\mu v}+q^{\mu} q^{v}\right]^{-1}$ which may be written in the form of $A g^{\mu v}+B q_{\mu} q_{v}$. The values of the constants $A$ and $B$ are found from the matrix identity $M M^{-1}=1$, hence, $\left[\left(-q^{2}+M^{2}\right) g^{\mu v}+q^{\mu} q^{v}\right]\left(A g^{\mu v}+B q_{v} q_{p}\right)=\delta_{p}^{\mu}$. Then, $A=-1 /\left(q^{2}-M^{2}\right)$ and $B=1 / M^{2}\left(q^{2}-M^{2}\right)$. This leads to the propagator form $\frac{-g^{\mu v}+q^{\mu} q^{v} / M^{2}}{q^{2}-M^{2}}$. Then the total transition matrix element has the form:

$$
M=J_{\mu}^{w k}\left[\frac{-g^{\mu v}+q^{\mu} q^{v} / M^{2}}{q^{2}-M^{2}}\right] J_{v}^{w k}
$$

Furthermore, a series of celebrated experiments [12-14] have shown that neutrinos have the following properties:

1) They are massless or nearly so in the standard model viewpoint.

2) There are three distinct types of neutrino each is associated with its own charged lepton: $\left(\mathrm{e}^{-}, v_{\mathrm{e}}\right),\left(\mu^{-}, v_{\mu}\right)$ and $\left(\tau^{-}, v_{\tau}\right)$.

3) They have spin $1 / 2$ but only the negative helicity state (left-handed) participates in weak interactions.

4) The weak interactions don't conserve $P$, the parity, not do they respect invariance under the charge conjugation. 
The study of the Lorentz covariance of Dirac equations defines the vector current as $\bar{u} \gamma_{\mu} u$ and the axial vector current as $\bar{u} \gamma_{\mu} \gamma_{5} u$, where $u$ is a 4-component wave function and $\gamma_{5}$ is a $4 \times 4$ matrix that defined in terms of the Dirac $\gamma$ matrices as: $\gamma_{5}=i \gamma^{0} \gamma^{1} \gamma^{2} \gamma^{3}$. Writing the parity operator $\mathrm{P}$ in the form $P=\left(\begin{array}{cc}1 & 0 \\ 0 & -1\end{array}\right)$, then it is possible to show that the vector current $V_{\mu}=\bar{u} \gamma_{\mu} u$ transforms under parity as $\bar{V}_{\mu_{-}}=\bar{u} \gamma_{0} \gamma_{\mu} \gamma_{0} u=\left(V_{0},-V\right)$, while the axial vector $A_{\mu}=u \gamma_{\mu} \gamma_{5} u$ transforms to $\bar{A}_{\mu}=\bar{u} \gamma_{0} \gamma_{\mu} \gamma_{5} \gamma_{0} u$, or in other words $\bar{A}_{\mu}=\left(-A_{0},+A\right)$.

The right and left-handed helicity operators $\mathrm{P}_{\mathrm{R}}, \mathrm{P}_{\mathrm{L}}$ are defined as $P_{R}=\left(\frac{1+\gamma_{5}}{2}\right)$ and $P_{L}=\left(\frac{1-\gamma_{5}}{2}\right)$, which satisfy the relations, $P_{L}^{2}=P_{L}, P_{R}^{2}=P_{R}, P_{L} P_{R}=P_{R} P_{L}=0$, $P_{L}+P_{R}=1$. So that, for massless spin $1 / 2$ neutrinos, the combination $\left(1-\gamma_{5}\right) u_{v}(p)$ contains only a left-handed component.

Instead of pure vector currents, we have now the vector $\mathrm{V}$, and axial vector, $\mathrm{A}$, pieces. The leptonic weak current for each lepton and its neutrino has the form,

$$
\begin{aligned}
J\left(v^{\prime} v\right) & =\left\langle v^{\prime}\left|J_{\mu}^{w k}\right| v\right\rangle \\
& =(g / \sqrt{2}) N N^{\prime} \bar{u}\left(v^{\prime}\right) \gamma_{\mu} \frac{1}{2}\left(1-\gamma_{5}\right) u(v)
\end{aligned}
$$

where $\mathrm{g}$, is the coupling constant for the $\mathrm{W}(\mathrm{Z})$ boson that exchanges in weak processes. The total interaction matrix element contains both the leptonic current and the hadronic or the quark current, written as:

$$
\begin{aligned}
& M=\left(g^{2} / 2\right) \bar{u}\left(v^{\prime}\right) \gamma_{\mu} \frac{1}{2}\left(1-\gamma_{5}\right) u(v) \frac{\left(-g^{\mu v}+q^{\mu} q^{v} / M_{Z}^{2}\right)}{q^{2}-M_{Z}^{2}} \\
& \sum_{i \neq j} \bar{u}\left(q_{j}{ }^{\prime}\right) \gamma_{\mu} \frac{1}{2}\left(1-\gamma_{5}\right) u\left(q_{i}\right)
\end{aligned}
$$

where q refers to the quark type $\mathrm{u}, \mathrm{d}, \mathrm{s}, \ldots$.

\section{Problem Statement}

A model for weak interaction of neutrino with nucleons is proposed. In this model we assume that the neutrino interacts with nucleons through the IVB which may be the $\mathrm{W}$ or $\mathrm{Z}$ with effective mass about $80 \mathrm{GeV}$. The Feynman diagram as in Figure 1 represents the interaction.

The scattering amplitude is then calculated according to Equation (6). The implementation of this equation reveals two main problems. The first of them is latent in the calculation of neutrino wave function to calculate the weak leptonic current. The second one comes in calculating the quark hadronic current, which consequently

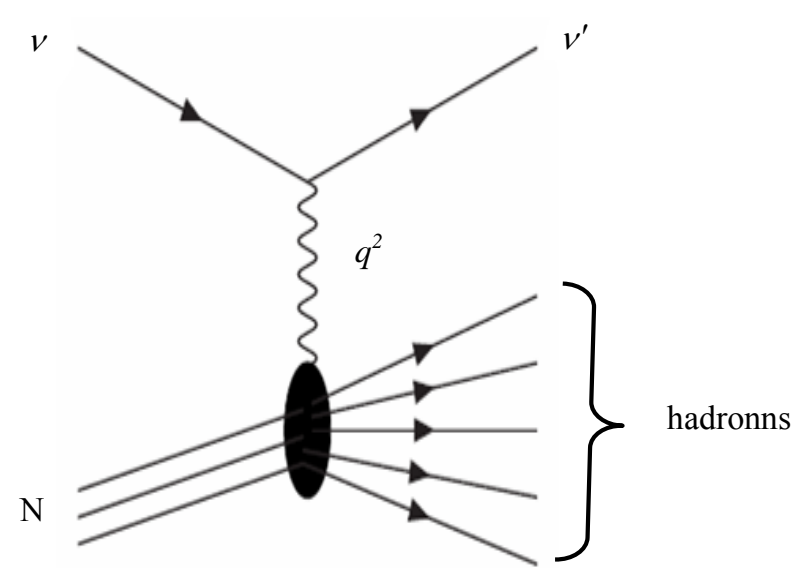

Figure 1. Feynman representation of the v-nucleon interaction.

needs the specification of the wave function of the quarks forming the nucleon.

\section{Results and Discusion}

As mentioned earlier Equation (5), the weak leptonic current is calculated as:

$$
\left\langle v^{\prime}\left|J_{\mu}^{w k}\right| v\right\rangle=(g / \sqrt{2}) N N^{\prime} \bar{\psi}\left(v^{\prime}\right) \gamma_{\mu} \frac{1}{2}\left(1-\gamma_{5}\right) \psi(v)
$$

where $\psi(v) \& \bar{\psi}\left(v^{\prime}\right)$, are the neutrino wave functions before and after scattering at the first vertex of Figure 1.

As a good approximation, it is possible to consider the neutrino's wave function as a plane wave with the form:

$$
\Phi_{j}(r, t)=u_{j} e^{i(k . r-\omega t)}
$$

The 4-component matrix u describes the spin $1 / 2$ particle:

$$
u=\left(\begin{array}{c}
1 \\
0 \\
\frac{P_{z}}{E+m} \\
\left(\frac{P_{x}+i P_{y}}{E+m}\right)
\end{array}\right)
$$

Since the neutrino is massless and moves initially in the z-direction so, $u=\left(\begin{array}{l}1 \\ 0 \\ 1 \\ 0\end{array}\right)$.

On the other hand, we used the perturbation technique to find the scattered wave function of the neutrino as: 


$$
\begin{aligned}
& \Psi\left(k^{\prime}, r\right)=\left\{\Phi\left(k^{\prime}, r\right)+\right. \\
& \left.(2 \pi)^{3 / 2} f \frac{\exp \left(i k^{\prime} r\right)}{r}\right\}\left(\begin{array}{c}
1 \\
0 \\
\cos \theta \\
\sin \theta+i \sin \theta \sin \phi
\end{array}\right)
\end{aligned}
$$

Where $\theta, \phi$ and $k$ 'are the azimuthal, polar angles and the momentum of the scattered neutrino, $f$ is the scattering amplitude and $r$ is the distance from the scattering center. Since the scattering is due to weak field, then it is sufficient to consider only one term in the perturbation series.

$$
\begin{aligned}
& \Psi\left(k^{\prime}, r\right)=\left\{(2 \pi)^{-3 / 2} e^{-i k^{\prime} r \cos \theta}+\right. \\
& \left.(\pi / 2)^{1 / 2} \frac{g}{\left(M^{2}+q^{2}\right)} \frac{\exp \left(-i k^{\prime} r\right)}{r}\right\}\left(\begin{array}{c}
1 \\
0 \\
\cos \theta \\
\sin \theta+i \sin \theta \sin \varphi
\end{array}\right)
\end{aligned}
$$

Then the first component of the leptonic current $J_{x}$ is given by,

$$
\begin{gathered}
J_{x}=\int_{0}^{R} \int_{0}^{2 \pi} \int_{0}^{\pi}\left[\frac{-1+2 \cos \theta+\sin \theta+i \sin \theta \cos \phi}{8 \pi\left(M^{2}+q^{2}\right) r}\right] \\
g r^{2} \sin \theta d \theta d \phi d r
\end{gathered}
$$

The integrals in Equation (12) are due to the averaging of the current allover the available space inside the nucleon of radius $\mathrm{R}$.

$$
J_{x}=-g \frac{\left(-1+e^{i q R}-i q R\right)}{6 \pi q^{2}\left(M^{2}+q^{2}\right)} e^{-i q R}
$$

Similarly $J_{y}, J_{z}$ are found to be:

$$
\begin{aligned}
& J_{y}=g \frac{\left(i\left\{-1+e^{i q R}\right\}+q R\right)}{6 \pi q^{2}\left(M^{2}+q^{2}\right)} e^{-i q R} \\
& J_{z}=-g \frac{\left(-1+e^{i q R}-i q R\right)}{12 \pi q^{2}\left(M^{2}+q^{2}\right)} e^{-i q R}
\end{aligned}
$$

The weak leptonic current density is a complex function of the momentum transfer $q^{2}$, the imaginary part of which measures the absorption rate. The current components $\mathrm{J}_{\mathrm{x}}$ and $\mathrm{J}_{\mathrm{y}}$ are equal in the absolute values, due to the assumption of azimuthal symmetry of the problem. Figure 2 displays the current components $J_{x}$ and $J_{z}$, while the total leptonic current is displayed in Figure 3.

Appreciable values of the current are obtained near small $\mathrm{q}^{2}$. To proceed further, we shall determine the wave functions for the $u$ and $d$ quarks, forming the nucleon by

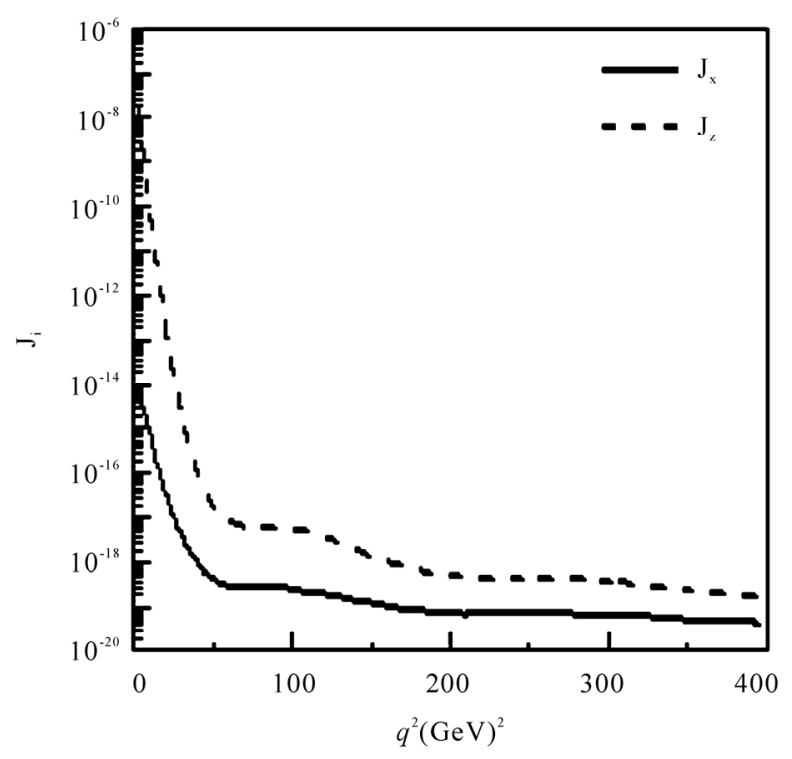

Figure 2. The lepton current components $J_{x}$ and $J_{z}$ as a function of $q^{2}$.

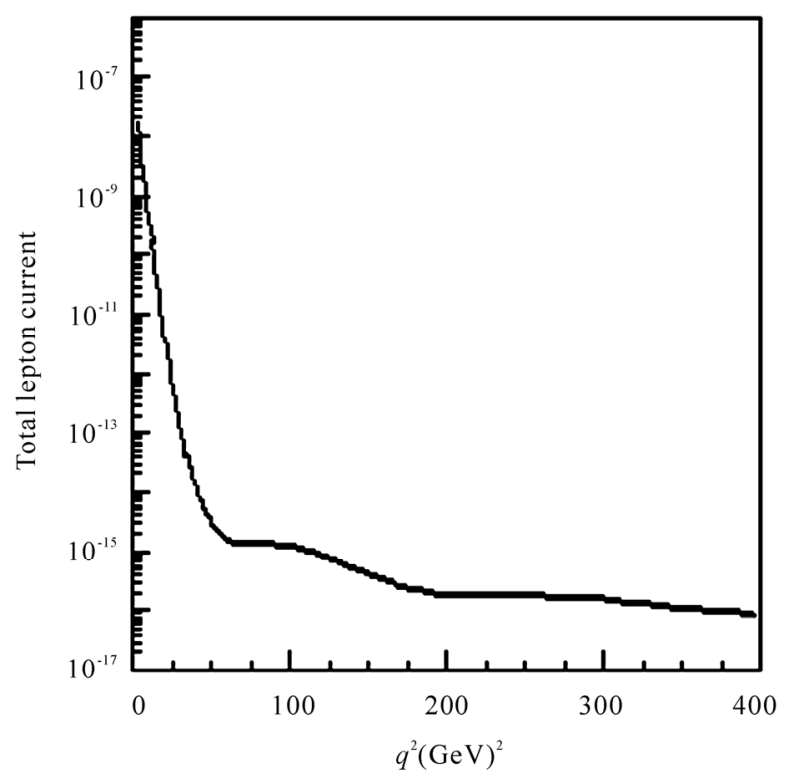

Figure 3. The total lepton current as a function of $q^{2}$.

empirical method. In other words, we shall use the values of the structure functions $\mathrm{F}_{2}(\mathrm{x})$ and $\mathrm{xF}_{3}(\mathrm{x})$ that extracted from the deep inelastic scattering of neutrino with nucleon. Making the approximation of setting the Cabibbo angle to zero, we obtain the correspondence

$$
\begin{aligned}
& F_{2}^{v p}=2 x[d(x)+\bar{u}(x)] \\
& F_{3}^{v p}=2 x[\bar{u}(x)-d(x)]
\end{aligned}
$$

where $F_{2}^{v p}$ and $F_{3}^{v p}$ are the structure functions for $v$-p scattering. Using the hadronic isospin invariance we get, 


$$
\begin{aligned}
& F_{2}^{v n}=2 x[\bar{d}(x)+u(x)] \\
& F_{3}^{v n}=2 x[\bar{d}(x)-u(x)]
\end{aligned}
$$

where $F_{2}^{v n}$ and $F_{3}^{v n}$ are the structure functions for $v-n$ scattering. Hence it is easy to define the quark and the anti-quark wave functions as:

$$
\begin{aligned}
& u(x)=\frac{F_{2}^{v n}-x F_{3}^{v n}}{4 x} \\
& \bar{u}(x)=\frac{F_{2}^{v p}+x F_{3}^{v p}}{4 x} \\
& d(x)=\frac{F_{2}^{v p}-x F_{3}^{v p}}{4 x} \\
& \bar{d}(x)=\frac{F_{2}^{v n}+x F_{3}^{v n}}{4 x}
\end{aligned}
$$

The structure function $\mathrm{F}_{2}$ and $\mathrm{xF}_{3}$ are functions only in the scaling variable $\mathrm{x}$ and approximately independent of the 4-momentum square $q^{2}$. The data of the experiments carried out in CERN-WA-025 [15] and FNAL-616 [16] are used to put the functions $\mathrm{F}_{2}$ and $\mathrm{xF}_{3}$ in parametric forms in the variable $x$, as shown by figures Figure 4 and Figure 5 for $v-n$ and $v-p$ reactions.

The structure function $\mathrm{F}_{2}$ is formulated as:

$$
\begin{aligned}
& F_{2}=1.99 \exp (-2.74 x) \text { for } v-n \\
& F_{2}=1.38 \exp (-3.95 x) \text { for } v-p
\end{aligned}
$$

while, the structure function $\mathrm{xF}_{3}$ is obtained as a polynomial of the $4^{\text {th }}$ order:

$$
\begin{gathered}
\mathrm{xF}_{3}=0.323+6.59 \mathrm{x}-16.93 \mathrm{x}^{2}+6.66 \mathrm{x}^{3}+5.06 \mathrm{x}^{4} \\
\text { for } \mathrm{v}-\mathrm{n} \\
\mathrm{xF}_{3}=0.153+3.28 \mathrm{x}-11.53 \mathrm{x}^{2}+12.03 \mathrm{x}^{3}-3.73 \mathrm{x}^{4} \\
\text { for } v-\mathrm{p}
\end{gathered}
$$

Figures $4 \& 5$ show that the structure function $F_{2}$ is more predominant in $v-n$ than the $v-p$ all over the range of $\mathrm{x}$. Their values are relatively close near the deep inelastic scattering $(\mathrm{x} \approx 0)$ and divert toward the elastic end $(\mathrm{x} \rightarrow 1)$. On the other hand the third structure function $\mathrm{xF}_{3}$ shows a bell shape in all cases with peak value near $(\mathrm{x} \approx$ $0.7)$. The $v-n$ structure function overpass that of $v-p$ with relatively constant ratio of 2 that divert to more than 4 near the elastic end. Accordingly we conclude that target constitution plays important role in the interaction cross section. In other words since neutrons are enriched with $\mathrm{d}$ quarks so a model that relies a point like interaction is much supporting collision of $v$-d more than $v$ collisions with u quarks. In this context we are able to extract the quark distribution functions $\mathrm{u}(\mathrm{x})$ and $\mathrm{d}(\mathrm{x})$ according to Equation (18).

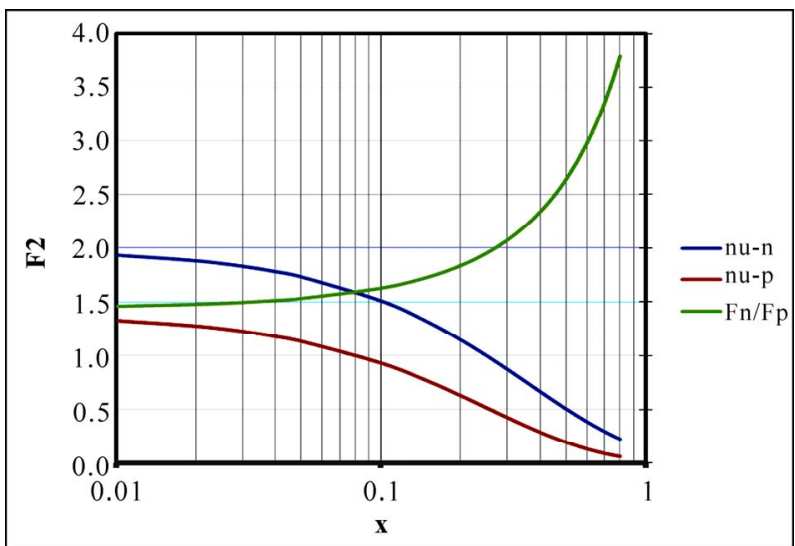

Figure 4. The relation between $F_{2}$ and $x$ for $v-p, v-n$ and their relative values.

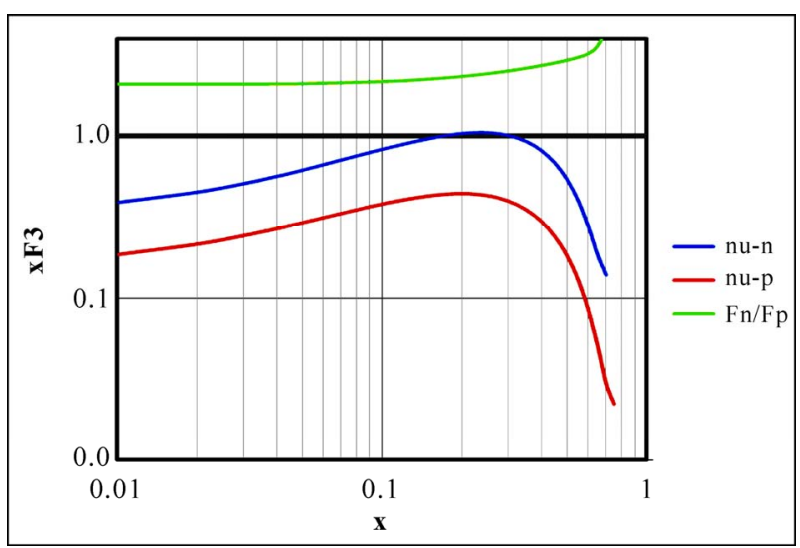

Figure 5. The relation between $\mathrm{xF}_{3}$ and $\mathrm{x}$ for $v-\mathrm{p}, v-\mathrm{n}$ and their relative values.

The quark functions $u, d, \bar{u}$ and $\bar{d}$ are calculated using Equation (18) and presented in Figure 6. It is clear that the quark wave functions have similar behavior with appreciable values only in the range $x<0.4$. Also, they are decreasing gradually with $\mathrm{x}$ and diminishes at $\mathrm{x}=1$. The quark current is then calculated tn terms of the quark wave function $u(\mathrm{u})$ and $u(\mathrm{~d})$

$$
J_{\text {quark }}=\sum_{i \neq j} \bar{u}\left(q_{j}{ }^{\prime}\right) \gamma_{\mu} \frac{1}{2}\left(1-\gamma_{5}\right) u\left(q_{i}\right)
$$

Figure 7 shows that the quark currents have minimum value in the range $0.4<\mathrm{x}<0.8$ for both $\mathrm{u}$ and d quarks, as well as they are very close to each other.

Further, according to Equation (6) the relation between the matrix element squared $\mathrm{M}^{2}$ and the momentum transfer square, $q^{2}$ is displayed in Figure 8 which reveals that the matrix element is almost independent on $q^{2}$ in the range $0.05<\mathrm{x}<0.5$. The general feature of the results seems comparable to those produced by CTEQ collaboration [17] and MRS collaboration [18] at adjacent energy values. 


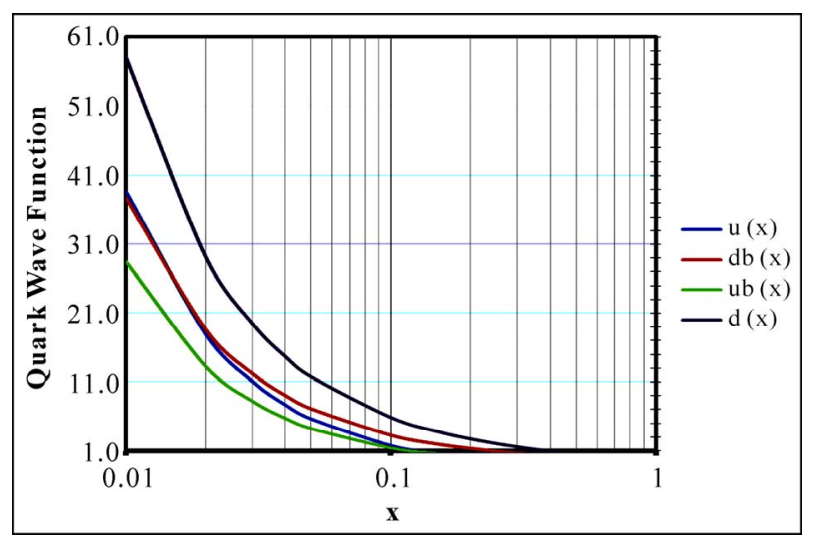

Figure 6. The wave functions of the quarks and antiquarks $u, d u$ and $d$ as estimated by the empirical method.

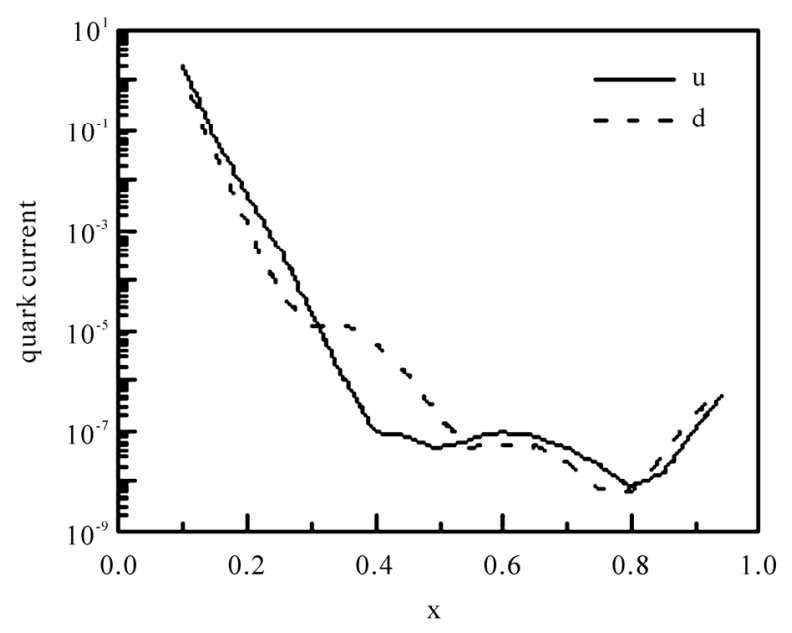

Figure 7. The weak quark current for $u$ and $d$ quarks as a function of $x$.

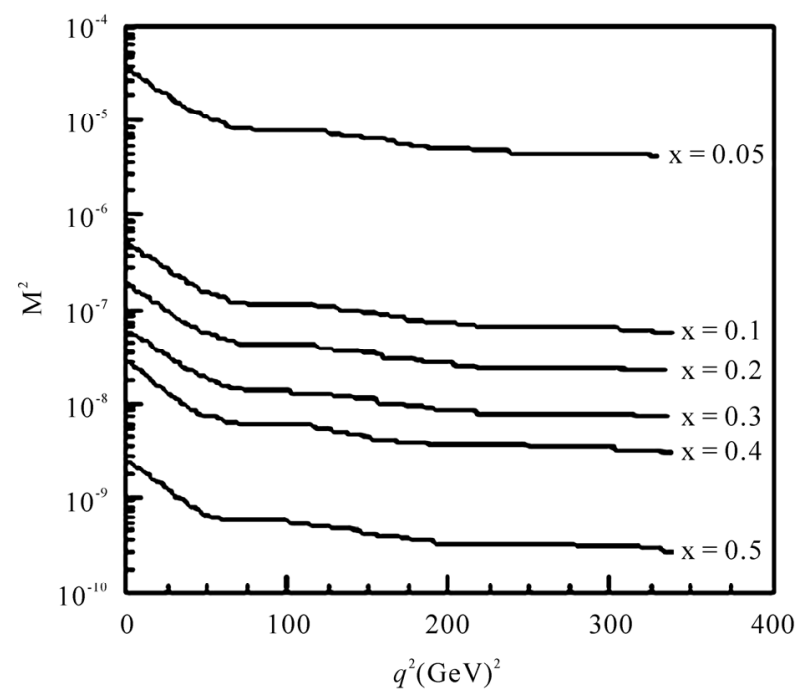

Figure 8. The matrix element squared as a function of $q^{2}$ at different $x$ values.

\section{Conclusions}

In summary, neutrino-nucleon interaction was investigated through intermediate vector boson (IVB). The neutrino wave function was derived with perturbed technique. Thus, the weak leptonic current can be obtained in term of $q^{2}$. Also, the quark wave functions were determined by empirical method based upon experimental data and the weak hadronic current can be estimated as a function of $\mathrm{x}$.

The differential deep inelastic cross section of neutrino-nucleon interaction is described in terms of three structure functions representing the three helicity states $H=1,-1$ and 0 . The appreciable increase of $v-n$ cross section compared to $v$-p supports the point particle interaction model and that $v$ is likely dependent on the quark flavor of the nucleon constituent quarks. The down quark $d$ structure function overpass that for the up quark $\mathrm{u}$ at all values of $\mathrm{x}$.

The quark distribution functions are also studied using $v$ [19], e and $\mu$ [20] inelastic scattering. The analyses are done in the leading order (LO) and next-to-leading order (NLO) of running coupling constant. Although the uncertainty by the NLO perturbation show slit modification at small $x$. however, they are not significant at larger $x$. In both cases the result of the analysis are very close to that obtained by the IVB model.

It is found also that the determination of the quark distribution functions are independent on the type of the projectile of the reaction whether it is $v$ or $\mathrm{e}$.

Moreover, the total interaction matrix element is calculated by IVB and NLO and found to be almost independent on $q^{2}$ in the range $0.05<\mathrm{x}<0.5$. The prediction of this analysis shows global fair agreement with experimental data in the neutrino energy range $\left(E_{v}\right)$ $150-250 \mathrm{GeV}$.

\section{References}

[1] J. L. Carr, arXiv: hep-ph/9903320.

[2] M. A. B. Beg and G. Feinberg, Physics Review Letters, No. 33, 1974, p. 606.

[3] T. Siiskonen et al., Physical Review C, No. 63, 2001, 055501 .

[4] J. Govaerts, Nuclear Instruments and Methods in Physics Research Section A, No. 402, 1998, p. 303.

[5] R. P. Feynman and M. Gell-Mann, Physical Review, No. 109, 1958, p. 193.

[6] M. L. Goldberger and S. B. Treiman, Physical Review, No. 110, 1958, p. 1178.

[7] G. Bardin et al., Physical Letters B, No. 104, 1981, p. 320.

[8] D. M. Wright et al., Physical Letters C, No. 57, 1998, p. 
373.

[9] T. Siiskonen et al., Physical Letters C, No. 59, 1999, p. 1839.

[10] T. Siiskonen et al, Journal of Physics G, No. 25, 1999, p. 55.

[11] B. Castel and I. S. Towner, "Modern Theories of Nuclear Moments", Clarendon Press, Oxford, 1990.

[12] K. Nishikawa, Conference of High Energy Physics Hep 2001, Budapest 2001, Invited talk.

[13] S. M. Bilenky, arXiv: hep-ph/0103091.

[14] D. H. Perkins, International Europhysics Conference HEP2001, Budapest 2001, Invited talk.
[15] A. G. Tenner and N. N. Nikolaev, Nuovo Cimento A, No. 105, 1992, p. 1001.

[16] R. Blair et al., Physical Review Letters, No. 51, 1983, p. 343.

[17] H. L. Lai et al., Physical Review D, No. 51, 1995, p. 4763.

[18] A. D. Martin et al., Physical Review D, No. 47, 1993, p. 867.

[19] A. Bodek and U. K. Yang, Nuclear Physics B, No. 112, 2002, p. 70.

[20] J. F. Owens et al., Physical Review D, No. 75, 2007, p. 054030 . 